\title{
EchoGéo
}

15 | 2011

La Belgique, État prospère en décomposition au cœur de l'Europe du nord-ouest

\section{La production de logements en Belgique et à Bruxelles - Acteurs, dynamiques, géographie}

\section{Christian Dessouroux et Alice Romainville}

\section{(2) OpenEdition}

\section{Journals}

Édition électronique

URL : https://journals.openedition.org/echogeo/12279

DOI : $10.4000 /$ echogeo. 12279

ISSN : 1963-1197

Éditeur

Pôle de recherche pour l'organisation et la diffusion de l'information géographique (CNRS UMR 8586)

Référence électronique

Christian Dessouroux et Alice Romainville, «La production de logements en Belgique et à Bruxelles Acteurs, dynamiques, géographie », EchoGéo [En ligne], 15 | 2011, mis en ligne le 11 avril 2011, consulté le 03 août 2021. URL : http://journals.openedition.org/echogeo/12279 ; DOI : https://doi.org/ 10.4000/echogeo.12279

Ce document a été généré automatiquement le 3 août 2021

EchoGéo est mis à disposition selon les termes de la licence Creative Commons Attribution - Pas d'Utilisation Commerciale - Pas de Modification 4.0 International (CC BY-NC-ND) 


\title{
La production de logements en Belgique et à Bruxelles - Acteurs, dynamiques, géographie
}

\author{
Christian Dessouroux et Alice Romainville
}

\section{Cinquante ans de production résidentielle en Belgique : une introduction}

1 La géographie des espaces résidentiels a évolué sensiblement au cours des dernières décennies en Belgique. La périurbanisation a repoussé les limites de la ville au point de les faire disparaître, l'habitat rural n'a plus la même signification. Les distances entre lieu de résidence et lieu de travail se sont de ce fait étirées considérablement et ce d'autant plus que l'Etat belge a très tôt soutenu la déconcentration urbaine et le mouvement pendulaire des travailleurs moyennant une politique d'accès à la propriété privée et d'amélioration des transports en commun d'abord, des infrastructures routières ensuite. Comparé aux autres pays européens, la périurbanisation a un caractère particulièrement soutenu et éclaté (Halleux et al., 2002), à mettre en relation avec une disponibilité abondante de terrains constructibles inscrits dans les plans d'affectation règlementaires et une gestion foncière faiblement planificatrice.

2 Les débuts de cette dispersion de l'habitat remontent à la fin du $19^{e}$ siècle et à l'introduction des premiers abonnements bon marché délivrés par les chemins de fer belges (Vandermotten, 2003), mais s'accélère considérablement depuis les années 1950. L'accession à la propriété est alors promue plus que jamais par un système de primes et de garanties de l'Etat afin d'encourager la construction de maisons modestes par les particuliers (loi dite De Taeye). Cette aide massive à la production privée, couplée à une démocratisation de l'accès à la voiture, constitue le moteur de la périurbanisation et alimente de nouvelles filières de production: la promotion immobilière privée et surtout la construction individuelle par autopromotion qui constitue une véritable spécificité belge (Halleux, 2005 ; illustration 1). C'est la maison individuelle (75\% du 
parc immobilier résidentiel) inscrite en banlieue urbaine qui répond au mieux aux aspirations des classes moyennes dans un contexte d'amélioration continue de la situation économique des ménages, de progression de leur pouvoir d'achat et de fort investissement de l'Etat dans le financement du logement (Mougenot, 1988 ; Dubois, 2002).

3 En revanche, l'habitat ancien, très présent dans les quartiers centraux et ouvriers des villes et dans les agglomérations industrielles, tombe en désuétude. Constitué avant tout de biens privés mis en location, ce parc se dégrade ou se réduit au fur et à mesure qu'une part des classes moyennes et aisées quitte les villes et que les projets spéculatifs se multiplient, particulièrement à Bruxelles. Une partie du parc privé locatif se transforme ainsi en logement résiduel (logement "social de fait») (De Lannoy \& Kesteloot, 1990; Charles, 2006) pour les couches populaires qui n'ont pas accès au nouveau marché de construction résidentielle, mais également pour les populations étrangères arrivées dès les années 1950. Ceci étant, une partie de la demande en logements bon marché est absorbée également par une politique renforcée de construction de logements sociaux (sous forme d'ensembles pavillonnaires et d'ensembles de barres et de tours de logements) des années 1950 aux années 1970 (Noël, 2009), au centre et aux périphéries des villes.

4 La transformation de l'environnement économique, suite à la crise qui se déclare dès le milieu des années 1970, va enclencher de profonds changements sur le marché de la production résidentielle. Peu enclins à investir, les pouvoirs publics et les acteurs privés font baisser drastiquement le volume de constructions neuves (illustration 1).

D'autres facteurs, d'ordre démographique cette fois-ci, vont influencer le cours des choses. La diminution de la taille et l'augmentation du nombre de ménages (décohabitation, divorce, personnes isolées, jeunes couples sans enfants), le prolongement du parcours d'accession des jeunes à l'autonomie familiale et financière ainsi que l'immigration étrangère continue vont faire croître progressivement la demande de logements en ville. L'offre répond à cette nouvelle demande en multipliant, dès les années 1990, les opérations immobilières en ville. En raison des moindres ressources foncières, le processus de transformation du bâti existant (subdivision, conversion en logement, rénovation lourde) va jouer un rôle fondamental, du moins dans les quartiers les plus anciens.

6 Le rôle des pouvoirs publics a changé également dans ce contexte. En continuant à investir dans la construction de logements, les pouvoirs publics orientent leurs actions également au profit d'une nouvelle aide à la personne, notamment dans les quartiers populaires des grandes villes où divers types de subsides et d'aides financières (contrats de quartier, politique des grandes villes, initiatives européennes) sont à disposition des habitants et pouvoirs locaux. L'ambition est d'améliorer la qualité du parc résidentiel existant et ce au détriment de la production, pourtant indispensable, d'une quantité de logements suffisante aux personnes les plus démunies, écartées de plus en plus du parc locatif privé en raison de l'enchérissement des prix locatifs et acquisitifs.

7 Ceci étant, la demande en logements neufs dans les banlieues résidentielles des villes a également repris après quelques années de crise, confirmant en cela la persistance du phénomène de périurbanisation accompagné, dès les années 1970, d'un certain desserrement des activités autour des zones urbaines, au profit de villes de taille moyenne ou de zones rurales voisines des grandes villes, et ceci malgré les effets 
négatifs de plus en plus aigus en termes de transport (coût et temps), d'environnement et de surcoûts publics en équipements collectifs et infrastructures routières.

Illustration 1 - Evolution du nombre de logements neufs dans des bâtiments résidentiels par filière de production

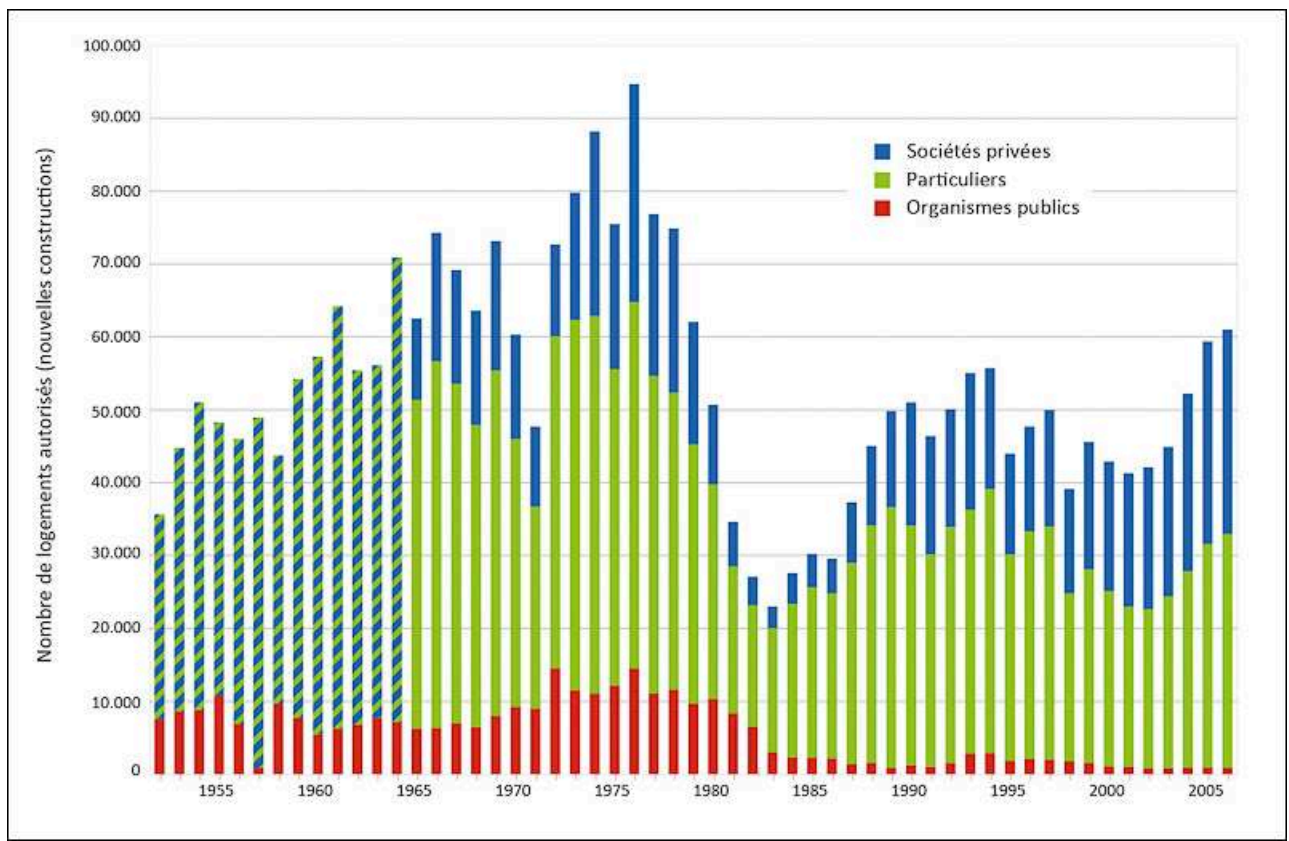

Source : Doucet, 1983 ; Direction Générale Statistique et Information économique ; Société du logement de la Région de Bruxelles-Capitale

8 Ces évolutions sont reflétées par les fluctuations du nombre de logements neufs autorisés par permis de bâtir chaque année (illustration 1) depuis 1952. Ce nombre est d'un peu plus de 50000 par an en moyenne ; on peut estimer que le parc de logements s'agrandit d'autant chaque année, et ce sans compter les logements créés par transformations, qu'elles soient légales ou illicites. Mais il y a de fortes variations d'une année à l'autre, selon des cycles essentiellement liés à la conjoncture économique et aux interventions de l'Etat. La périurbanisation, la politique du logement axée sur «l'aide à la pierre» et une rentabilité attrayante des investissements immobiliers assurent une activité croissante dans le secteur de la construction résidentielle entre l'après-guerre et la fin des années 1960. La construction passe par un maximum, en Belgique comme dans d'autres pays européens, dans les années 1970 (Louvot, 2001).Mais la crise s'annonce déjà au milieu des années 1970 avec la montée du chômage et le nombre de logements construits commence à diminuer pour connaître un minimum historique en 1983: c'est que les politiques de restriction salariale provoquent une contraction de la demande solvable (acquéreurs et locataires), contraction qui menace les débouchés de l'investissement immobilier. S'ajoutent à cela un taux hypothécaire élevé à l'époque, un relèvement des taux d'intérêt à court terme qui rend progressivement les placements mobiliers plus attrayants que les investissements immobiliers, et le blocage des loyers - conçu en 1975 comme une mesure temporaire pour compenser la compression des salaires - qui rend la propriété à bail financièrement moins profitable (voir aussi Doucet, 1983 ; De Decker, 2001). Le nombre de logements produits retrouve son niveau de 50000 unités annuelles en 1990 pour entamer un nouveau cycle (baisse jusqu'en 2000 environ, remontée ensuite) mais 
de moindre amplitude. L'autre évolution remarquable de ces 40 dernières années est la diminution drastique de l'intervention publique : la part des acteurs publics dans le nombre de logements produits, qui avait atteint $20 \%$ en 1972, puis $24 \%$ en 1981, est tombée à $1,5 \%$ en 2006 ! Les sociétés, à l'inverse, prennent une importance croissante sur le marché de la construction: de $25 \%$ environ dans les années 1970, elles assureraient aujourd'hui presque la moitié de la production ( $46 \%$ en 2006). Le grand nombre de propriétaires privés et l'importance de l'autopromotion a pu freiner, jusque dans les années 1980, l'apparition massive des promoteurs professionnels sur ce marché - alors qu'ils sont apparus bien plus tôt en France, par exemple (Topalov, 1974 ; Doucet, 1983). Avec le désengagement de l'Etat, et le marché devenu moins accessible aux particuliers, les sociétés (principalement des promoteurs immobiliers) ont pu augmenter radicalement leur présence sur le marché du logement belge.

\section{La production récente de logements en Belgique}

En 2008, la Belgique compte environ 4,6 millions de logements ${ }^{1}$ abritant une population d'un peu moins de 10,7 millions d'habitants (6,16 en Flandre, 3,46 en Wallonie et 1,05 à Bruxelles). Le taux d'urbanisation (plus de $20 \%^{2}$ ) et la densité de population (350 habitants par $\mathrm{km}^{2}$ ) élevés sont caractéristiques de l'Europe du Nord-Ouest.

Illustration 2 - La densité de population par commune en Belgique (2007) - Carte de repérage

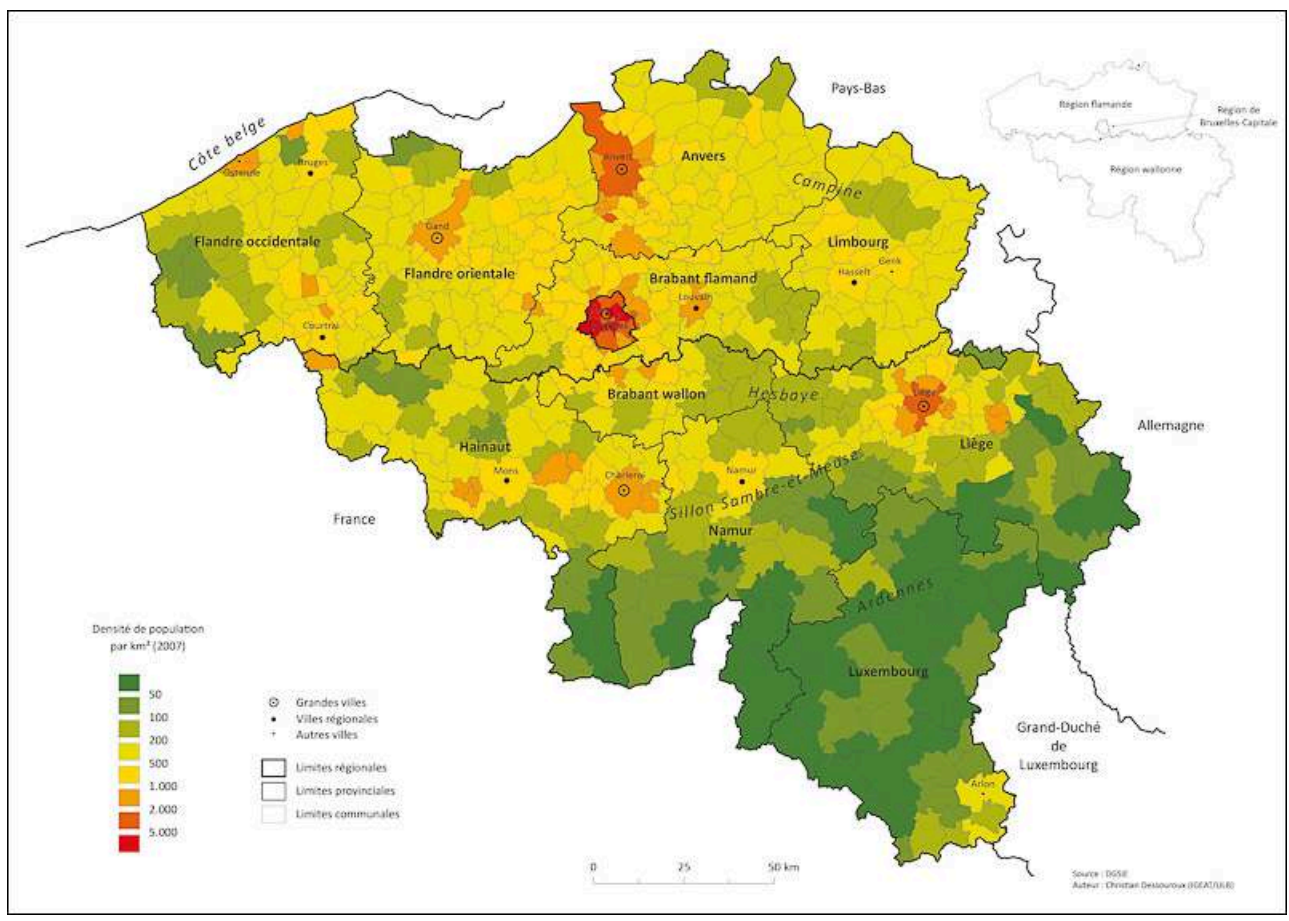

Source : DGSIE - Cartographie : Ch. Dessouroux (IGEAT/ULB).

10 La carte 2 permet de distinguer les deux principales zones de forte densité : le triangle Bruxelles-Anvers-Gand qui se prolonge à l'est vers le Limbourg et au sud vers le Brabant wallon; le sillon Sambre-et-Meuse constitué par les zones urbaines et industrielles wallonnes. D'autres pôles de forte densité existent dans la région de Courtrai, autour du bipôle Hasselt-Genk et le long de la côte. La périurbanisation, 
particulièrement lâche et consommatrice d'espace en Belgique, maintient les densités à un niveau encore modéré en regard de la taille de la population, notamment si on les compare à celles de certaines régions du Pays-Bas où la densification du bâti est plus forte et l'étalement urbain moindre. Seules les zones rurales situées au sud du sillon wallon et l'ouest de la Flandre ont des densités de population nettement moins élevées.

\section{L'état des données}

11 L'article a comme objectif premier d'analyser les évolutions de la géographie de l'offre en logements depuis une quinzaine d'années. Plutôt que d'étudier l'état du parc de logements, la structure des ménages ou l'évolution de la demande, nous nous intéressons à la production, aux projets immobiliers, à leur nature et à leur géographie. A cet effet, le recours à des données détaillées, nommément celles relatives aux permis de bâtir, s'impose. Tout projet immobilier nécessite en effet l'octroi préalable d'une autorisation de la part des autorités publiques compétentes. La Direction générale Statistique et Information économique (DGSIE) centralise l'ensemble des formulaires statistiques relatifs aux permis accordés. Depuis 1996, les données relatives au nombre de logements autorisés, à leur superficie et au mode de production (nouvelle construction, transformation) sont disponibles sur support informatique à l'échelle des communes. La présente contribution donne un panorama de résultats commentés issus de ces statistiques générales.

Précisons encore que l'évolution du nombre de logements est la résultante des créations de nouvelles unités et des disparitions. Hormis les constructions neuves, il y a création de nouvelles unités par division de logements existants ou par la conversion d'immeubles non résidentiels en logements. La disparition de certaines unités est due aux démolitions, aux fusions de plusieurs logements et aux transformations en locaux à usage autre que résidentiel. Les données issues de la DGSIE ne permettent toutefois pas de différencier les différents types de transformations. Le bilan net de logements autorisés, utilisé comme principale donnée dans les cartes, correspond à la différence entre le nombre de logements supplémentaires autorisés et le nombre de logements dont la suppression a été autorisée par permis de bâtir, que la création ou suppression de logement provienne d'une construction neuve / démolition ou de la transformation d'un bâtiment existant. Ce bilan général constitue un bon indicateur de la production de logements, malgré le fait que certains logements autorisés ne soient finalement pas produits et que des logements soient encore produits ou supprimés de manière illicite (sans permis préalable).

Dans le but d'étudier, dans un deuxième temps, les transformations du marché de production résidentielle à une échelle plus fine que l'entité communale, le recours à des données plus détaillées a été nécessaire. Les problèmes de disponibilité de données à l'échelle infracommunale imposent néanmoins de travailler sur un espace plus restreint que la Belgique et une période plus courte. Le choix a porté sur la Région de Bruxelles-Capitale dont des données par permis individuel nous ont été mises à disposition par le Ministère de la Région de Bruxelles-Capitale ${ }^{3}$ pour la période 2003-2008. L'étude du cas de Bruxelles, à la fois capitale nationale et européenne, se justifie d'autant plus que la ville vit une période de croît démographique, récent mais soutenu, s'inscrivant dans le contexte d'une politique publique volontariste de 
« revitalisation » urbaine, et une crise du logement aiguë qui touche avant tout les populations défavorisées et qui expose la ville à d'importants défis sociaux.

\section{Géographie de la production de logements en Belgique}

Deux modes de visualisation cartographique ont été choisis pour décrire l'évolution du nombre de logements: une représentation sous forme de cercles de taille proportionnelle au nombre de logements supplémentaires autorisés de 1996 à 2008 et une sous forme de taux d'accroissement annuel moyen.

Illustration 3 - Bilan net de logements autorisés par commune en Belgique (1996-2008)

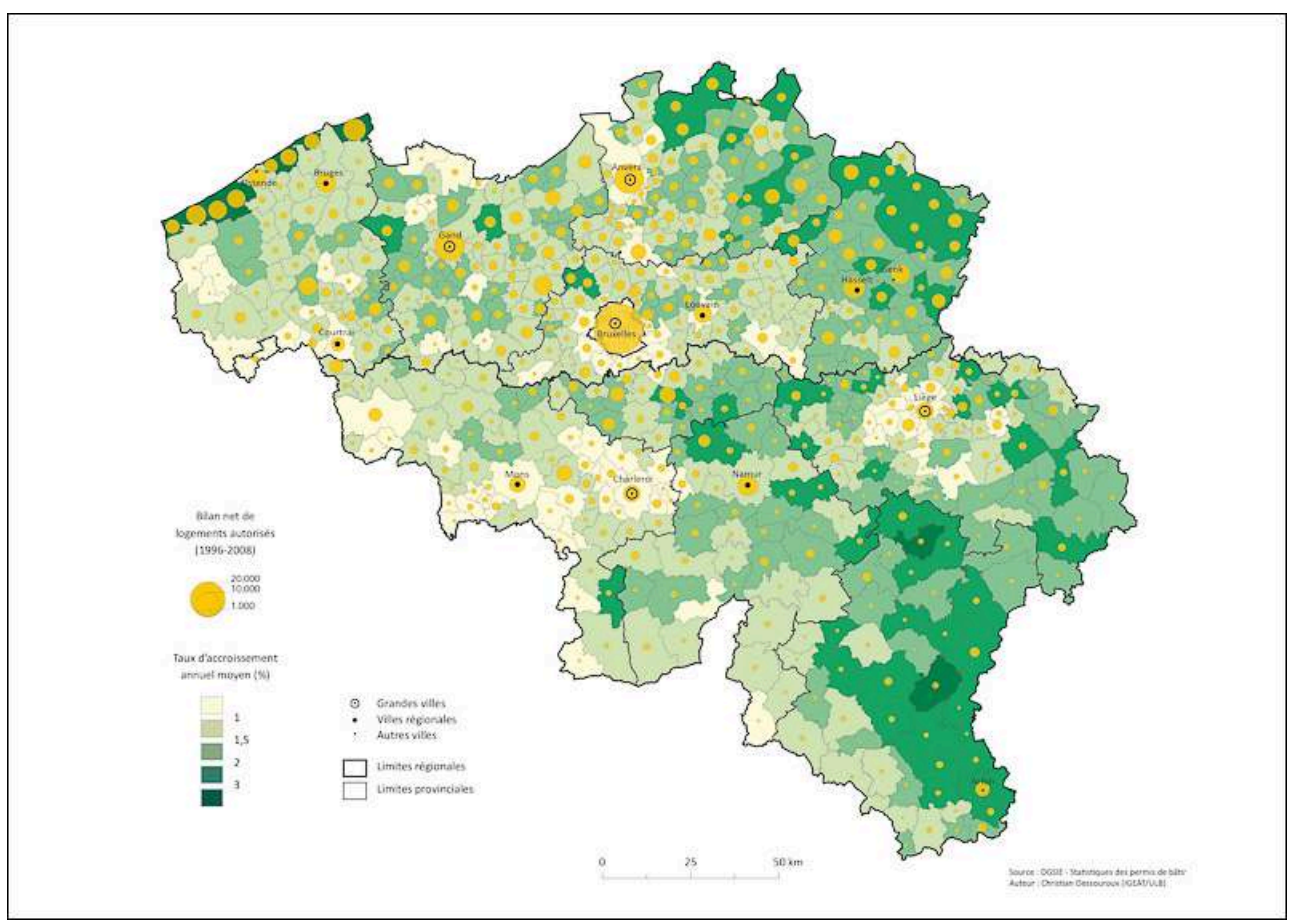

Source : DGSIE Statistiques de permis de bâtir - Cartographie : Ch. Dessouroux (IGEAT/ULB)

La lecture, en valeurs absolues, du bilan net de logements autorisés par commune en Belgique donne un premier aperçu des variations spatiales de la dynamique de production. Les logements neufs étant destinés à accueillir de nouveaux ménages ou à remplacer les logements devenus trop vétustes, la production est corrélée largement à la densité du bâti existant et à la structure démographique. Le nombre de projets immobiliers est dès lors globalement plus élevé dans les zones centrales et périphériques des grandes villes comme Bruxelles, Gand, Anvers, Namur et Liège, mais également, de manière plus générale, en Région flamande ainsi que le long de la côte belge. Cette dernière, mais également, plus discrètement, certaines communes rurales touristiques des Ardennes, exercent un attrait particulier à la fois comme lieu de seconde résidence ou lieu de vacances et comme domicile fixe pour une population retraitée.

16 Le phénomène de périurbanisation, particulièrement précoce et prononcé en Belgique, se poursuit indiscutablement comme en témoigne le nombre élevé de nouvelles unités résidentielles autorisées autour des grandes villes. Les régions situées au sud du sillon Sambre-et-Meuse (hormis les zones à proximité du pôle d'emploi de la ville de 
Luxembourg)et l'arrière-pays côtier enregistrent, en revanche, une activité relativement faible en chiffres absolus.

Pour mieux apprécier les contrastes régionaux en termes d'activité de production et éliminer l'effet qu'exerce la taille des communes et leur population sur le nombre de projets de logement, nous avons fait le choix d'utiliser un autre indicateur: le taux d'accroissement annuel du parc immobilier résidentiel, exprimé en \%. Le stock de logements initial est approché sur base du nombre de ménages inscrits au registre de population.

On constate en premier lieu que l'évolution du nombre de logements est positive partout en Belgique. La période de désinvestissement des agglomérations wallonnes (Liège, Charleroi) et de Bruxelles, très marquée dans les années 1980 encore, est révolue. Ce sont néanmoins toujours les grandes agglomérations et les villes régionales qui enregistrent les taux les moins élevés. La moindre disponibilité de terrains à bâtir et la difficulté d'y accéder à une offre immobilière qui soit à la fois de qualité et financièrement accessible expliquent évidemment le contraste persistant entre les communes urbaines et les communes périurbaines et rurales.

L'étalement urbain se poursuit quant à lui autour des villes belges. Il se nourrit toujours d'une aspiration des Belges à la maison individuelle, préférentiellement sous forme d'unité pavillonnaire ou jumelée, et de la quête d'un espace au cadre de vie attrayant et financièrement abordable. Mais la périurbanisation est désormais reportée vers des communes plus éloignées des grandes villes. Ce sont les communes de seconde périphérie, aux densités de population plus faibles, aux prix fonciers moins élevés et aux réserves de terrains constructibles plus nombreuses, qui ont connu les taux les plus élevés. Le chemin de fer, mais également les autoroutes, ont été les principaux vecteurs de cette extension des espaces résidentiels qui perdure malgré les coûts économiques et environnementaux de la périurbanisation et malgré l'élaboration de schémas d'orientation à l'échelle régionale ${ }^{4}$ pour freiner la dissémination de l'habitat dans les zones rurales. En l'absence d'outils d'action efficaces au niveau local ${ }^{5}$, la périurbanisation se poursuit donc, également encouragée par le morcellement de la propriété, les pratiques spéculatives (Dubois, 2002), la fiscalité immobilière (plus élevée dans les communes urbaines) et la décentralisation des compétences fiscales qui encourage les communes à vouloir sans cesse attirer de nouveaux habitants (Dubois, 2004 ; Romainville, 2007).

Si les communes rurales enregistrent des taux d'accroissement globalement élevés, trois zones se démarquent. La Campine limbourgeoise confirme ses taux élevés liés à une natalité relativement forte, à une périurbanisation soutenue et à la migration venue des Pays-Bas (Willems, 2008). La région de la Hesbaye, située entre Bruxelles, Namur et Liège, profite grandement de l'arrivée de familles de travailleurs qualifiés occupés dans les villes proches (Eggerickx et al., 2007). Et les taux particulièrement élevés des zones ardennaises sont liés notamment à leur meilleure accessibilité suite à l'extension du réseau autoroutier dans les années 1980. Au sein de cet espace, il y a lieu de distinguer d'une part une activité de construction liée à l'important mouvement migratoire de personnes en âge de retraite vers les communes ardennaises plutôt centrales et d'autre part le dynamisme démographique des communes situées le long de la frontière avec le Grand-Duché de Luxembourg (Capron et al., 2002). Cet espace transfrontalier profite beaucoup de son appartenance au bassin de main-d'œuvre de la ville de Luxembourg, pôle de développement tertiaire et principal centre d'emploi de la 
région. L'accroissement de la population, et celui de la demande en logements, y sont dus à la venue d'une main d'œuvre jeune et qualifiée qui se distingue également par ses taux de natalité relativement élevés.

21 Si cette analyse met en avant les contrastes géographiques à l'échelle de la Belgique, elle reste muette sur un point primordial : la production globalement insuffisante de logements observée dans chacune des trois Régions belges (Gouvernement wallon, 1999 ; De Decker, 2001).

22 C'est ainsi que l'augmentation récente de la population bruxelloise, à titre d'exemple, n'est pas compensée par une création proportionnelle de logements. 3200 logements ont en moyenne été créés entre 1996 et 2008 alors que le nombre de ménages a augmenté annuellement de quelque 4000 unités. La situation risque dès lors de continuer à se dégrader et ce particulièrement pour les populations les plus démunies.

23 Si la demande en logement stimule (insuffisamment) la réalisation de nouvelles constructions, elle favorise également la valorisation par transformation des espaces bâtis existants, qu'ils soient résidentiels ou non au départ.

\section{Une part croissante issue de la transformation de bâtiments existants}

La création de nouveaux logements résulte à la fois de la construction d'habitations neuves et de la mutation du parc immobilier existant (reconversion, agrandissement, subdivision...). L'illustration 4, qui nous renseigne sur l'évolution annuelle de la part des transformations dans la production globale de logements, montre à quel point les opérations de transformation du bâti existant ont gagné en importance dans la production globale de logements. Leur part tend à augmenter partout en Belgique et plus particulièrement en Région de Bruxelles-Capitale, où elle atteint désormais presque un tiers de la production totale. 
Illustration 4 - Variation de la part des transformations dans le bilan global net de logements autorisés, par Région (1996-2008)

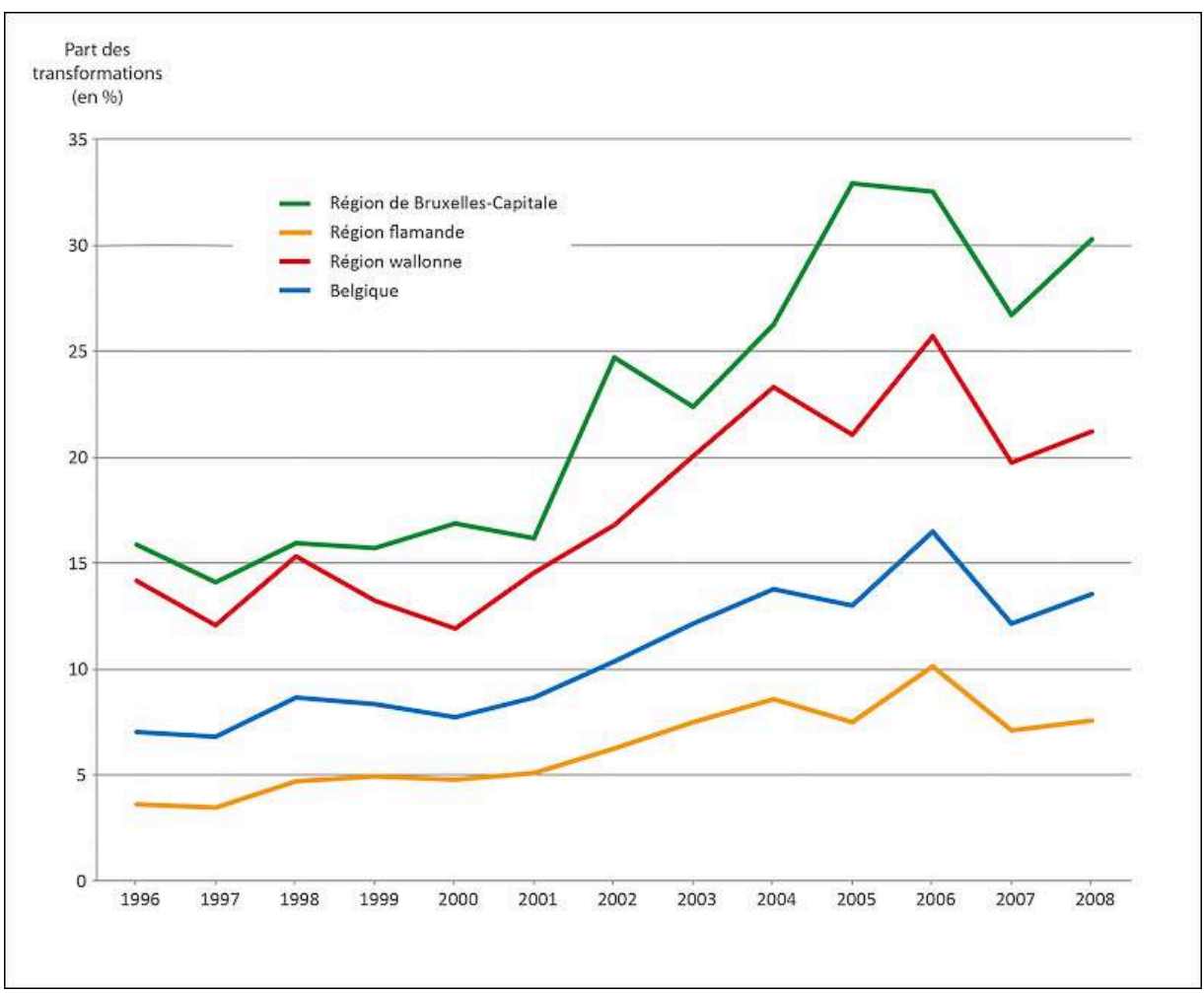

La question du logement et de sa production se pose donc à présent dans de nouveaux termes, à savoir à travers le développement notable du marché immobilier de l'ancien. Cette évolution est à mettre en rapport avec l'émergence, dès les années 1970, d'une politique d'amélioration de l'habitat et de l'environnement urbain dans les zones résidentielles désinvesties (aujourd'hui levée au rang d'axe stratégique de la politique de développement urbain). Celle-ci prend largement appui sur la valorisation et la réappropriation des zones urbaines (et rurales) disposant d'un patrimoine immobilier ancien par une population composée avant tout de petits ménages de jeunes adultes qualifiés (Noël, 1998 ; Van Criekingen, Decroly, 2003). Ce mouvement alimente à la fois les opérations d'auto-réhabilitation par les (nouveaux) propriétaires-occupants et les investissements des promoteurs privés (d'où également une relance de la construction neuve privée dans ces quartiers longtemps délaissés). 


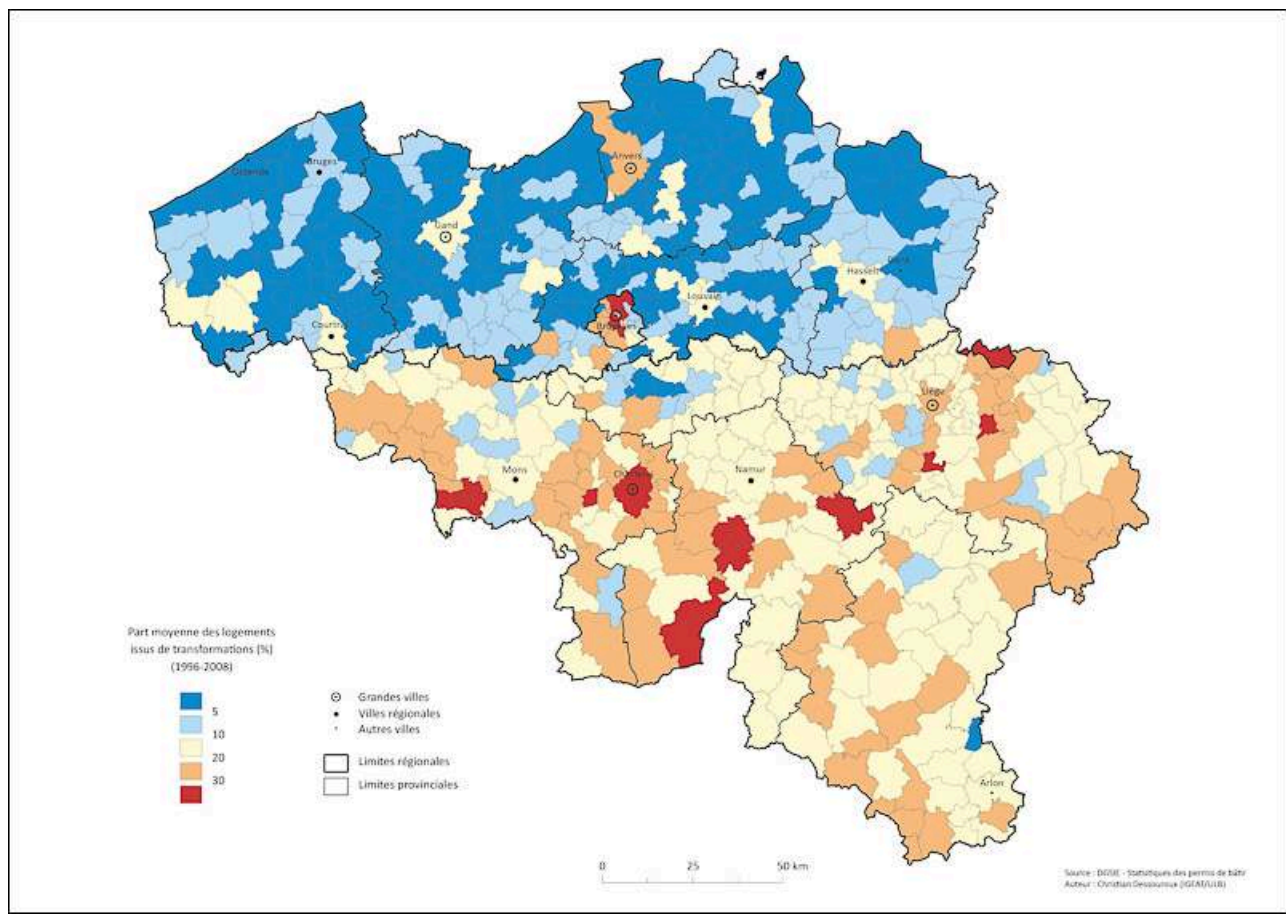

Source : DGSIE Statistiques de permis de bâtir -Cartographie : Ch. Dessouroux (IGEAT/ULB).

En étudiant l'illustration 5, on constate de prime abord que la part de transformations est, de manière générale, plus faible dans le nord que dans le sud du pays. Cette différentiation géographique régionale est constatée au moins depuis les années 1980 (Goossens et al., 1999) et se confirme donc pour la période actuelle. Plusieurs facteurs aident à interpréter ce résultat. La Flandre a tout d'abord connu, depuis la Deuxième Guerre mondiale, une activité de construction très importante, liée à son évolution économique particulièrement favorable, renouvelant une bonne partie du stock de logements existants. C'est particulièrement vrai pour les zones rurales mais également pour la zone côtière où l'établissement de domiciles fixes s'associe à une intense urbanisation touristique de résidences secondaires. Il n'empêche que dans ces zones densément peuplées, le nombre absolu de transformations soit élevé. Mais la part relative est en moyenne sensiblement moindre.

Les grandes villes comme Bruxelles, ainsi que le sud du pays possèdent, en revanche, un parc immobilier globalement plus ancien, hérité de la période d'industrialisation et d'urbanisation du $19^{e}$ et de la première moitié du $20^{\mathrm{e}}$ siècle. Construits pour une bonne part avant $1945^{6}$, ces logements sont plus souvent en mauvais état et insuffisamment équipés. Ils nécessitent généralement des améliorations structurelles et qualitatives importantes et sont dès lors davantage sujets à des transformations plutôt qu'à des rénovations légères.

Les communes wallonnes situées en dehors de l'axe industriel possèdent, plus spécifiquement, un stock important d'espaces bâtis affectés autrefois à l'activité agricole. Etant traditionnellement de plus grande taille et de meilleure qualité que l'habitation rurale flamande, les bâtiments ont souvent échappé à la démolition et se prêtent bien à une conversion. 
Les zones urbaines offrent une série d'opportunités de transformation complémentaires : outre la présence d'un important stock de maisons anciennes, certaines villes disposent également d'un nombre significatif de bâtiments non résidentiels abandonnés suite à la désindustrialisation et offrant un potentiel de reconversion non négligeable.

L'offre surabondante de surfaces tertiaires et la demande importante en logements à Bruxelles ont déclenché par ailleurs une réappropriation résidentielle partielle de bâtiments occupés un temps par des bureaux voire construits comme immeubles de bureau (voir ci-dessus ; illustration 8). La part élevée des transformations s'explique sans doute aussi par le fait que la progression du nombre de ménages, due entre autres à l'immigration étrangère et à la décohabitation (diminution de la taille et augmentation du nombre de ménages), touche davantage les milieux urbains.

31 La Région de Bruxelles-Capitale, la plus importante des agglomérations belges, à la fois capitale fédérale et européenne, sera examinée ci-après de manière à identifier à la fois les spécificités de la production de logements en milieu urbain et les défis auxquels sont exposés les villes compte tenu de leur croissance démographique et de leur dualisation sociale.

\section{La production de logements dans la Région de Bruxelles-Capitale}

\section{Les éléments d'une crise persistante du logement}

Comme dans les autres agglomérations belges, et contrairement au reste du pays, la majorité du parc immobilier bruxellois appartient au secteur locatif: malgré une politique du logement fortement axée sur le soutien à la propriété, le taux de propriétaires y plafonne à $40 \%$ depuis le début des années $1990^{7}$, contre une proportion d'environ $75 \%$ pour l'ensemble du pays.

La Région de Bruxelles-Capitale connaît par ailleurs une augmentation de sa population depuis 1995, qui s'explique avant tout par l'immigration venue de l'étranger, que ce soit de familles de travailleurs peu qualifiés ou de ménages aisés, composés souvent de personnes isolées travaillant dans les entreprises et institutions internationales ou dans les services gravitant autour. Mais Bruxelles connaît aussi depuis 1985 un solde naturel positif vu sa structure d'âge rajeunie (Deboosere et al., 2009).

Cette évolution provoque une augmentation de la demande et une concurrence accrue sur le marché du logement, d'autant plus que la présence de travailleurs étrangers très qualifiés exerce une pression à la hausse sur les prix acquisitifs et locatifs (Biot \& Van Hamme, 2007).

Couplés à vingt ans d'augmentation continue des loyers et des prix d'achat, ces éléments ont créé, à Bruxelles, les bases d'une sérieuse crise du logement qui se matérialise par un parc de logements en assez mauvais état, une forte sur-occupation des logements dans les quartiers populaires (38\% des enfants bruxellois vivaient dans un logement sur-occupé en $2001^{8}$ ), et une part très importante des revenus des ménages consacrés au frais de logement - souvent au détriment d'autres postes budgétaires vitaux comme les soins de santé (Observatoire de la santé et du social, 2009). 

logement social, secteur très peu représenté à Bruxelles avec un parc de quelques 38000 logements seulement ( $8 \%$ des logements). Le logement social absorbe environ $40 \%$ du budget « politique de l'habitat » de la Région (Zimmer, 2006), mais ces dépenses servent principalement au maintien du stock existant; pour des raisons à la fois budgétaires et politiques (notamment de relations entre le pouvoir régional et les communes), ce stock ne s'accroît pratiquement plus : on n'a produit qu'une trentaine de logements sociaux par an au cours des années 2000.

Les problèmes de sur-occupation et de mauvaise qualité des logements touchent surtout les jeunes, les femmes et les personnes faiblement qualifiées (Surkyn et al., 2007). Vu la forte ségrégation socio-spatiale à Bruxelles (De Lannoy et Kesteloot, 1990), ils se concentrent également dans certains quartiers: la carte (illustration 6) fait ressortir les quartiers populaires du centre de la Région avec les proportions les plus élevées de logements en mauvais état (est de Molenbeek et d'Anderlecht, ouest de Saint-Gilles et du pentagone, Saint-Josse, ouest de Schaerbeek). Les autres zones qui ressortent sur la carte avec des proportions élevées sont les quartiers périphériques où se trouvent des cités de logements sociaux en piteux état.

Illustration 6 - Logements en mauvais état (2001)

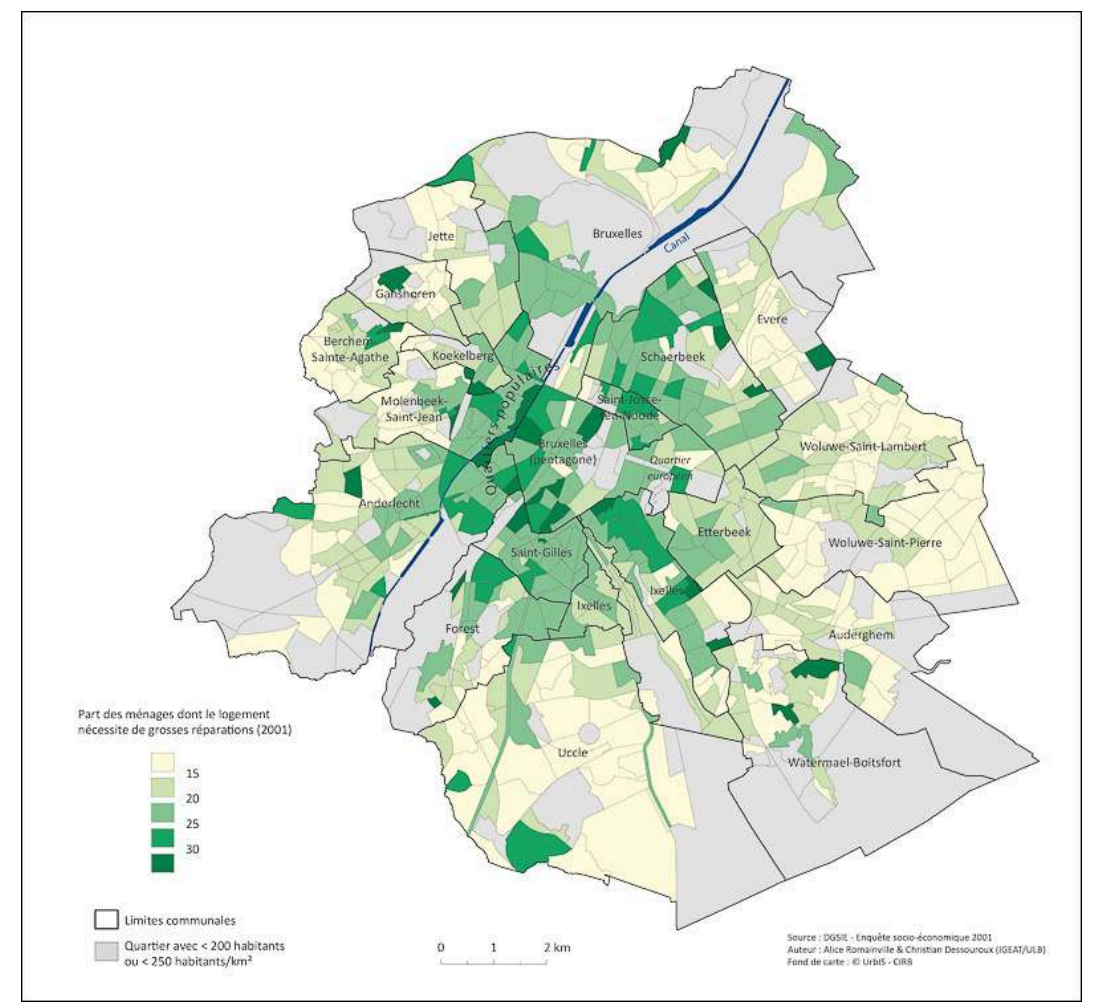

Source : DGSIE Enquête socio-économique - Cartographie : A. Romainville et Ch. Dessouroux (IGEAT/ ILB) ; fond de carte : (c) urbIS-CIRB.

Cette géographie évolue : depuis les années 1980, une partie des quartiers centraux et de première couronne connaissent des processus d'embourgeoisement et de gentrification, à travers des rénovations de l'environnement bâti et l'arrivée d'une population nouvelle et plus aisée (De Lannoy et Kesteloot, 1990 ; Van Criekingen, 2006). Un turnover très rapide de la population et l'absence de régulation sur le marché du 
logement y permettent une hausse rapide des loyers. Cette augmentation des prix oblige les habitants en place, lorsqu'ils sont amenés à déménager, à consacrer une part plus importante de leur revenu aux frais de logement ou à quitter le quartier ; de façon générale, elle rend le quartier moins accessible aux classes populaires (Van Criekingen, 2009). Les processus de revalorisation immobilière que connaissent les quartiers centraux renforcent donc, de diverses façons, la ségrégation socio-spatiale à l'échelle de la ville.

\section{Géographie de la production}

Les statistiques publiées par la DGSIE nous ont permis d'étudier l'évolution de la production en Belgique depuis 1996. Etant donné que ces données ne sont disponibles qu'à l'échelle communale, nous avons eu recours à des données plus détaillées, par quartier, pour Bruxelles. Celles-ci sont collectées et encodées par l'administration régionale (Administration de l'Aménagement du Territoire et du Logement (AATL) du Ministère de la Région de Bruxelles-Capitale ${ }^{9}$ ) et disponibles, de manière exhaustive, pour la période 2003-2008 seulement. Elles sont néanmoins représentatives des changements observés depuis le milieu des années 1990 (Ananian, 2010).

Illustration 7 - Bilan global net de logements autorisés à Bruxelles, par quartier (2003-2008)

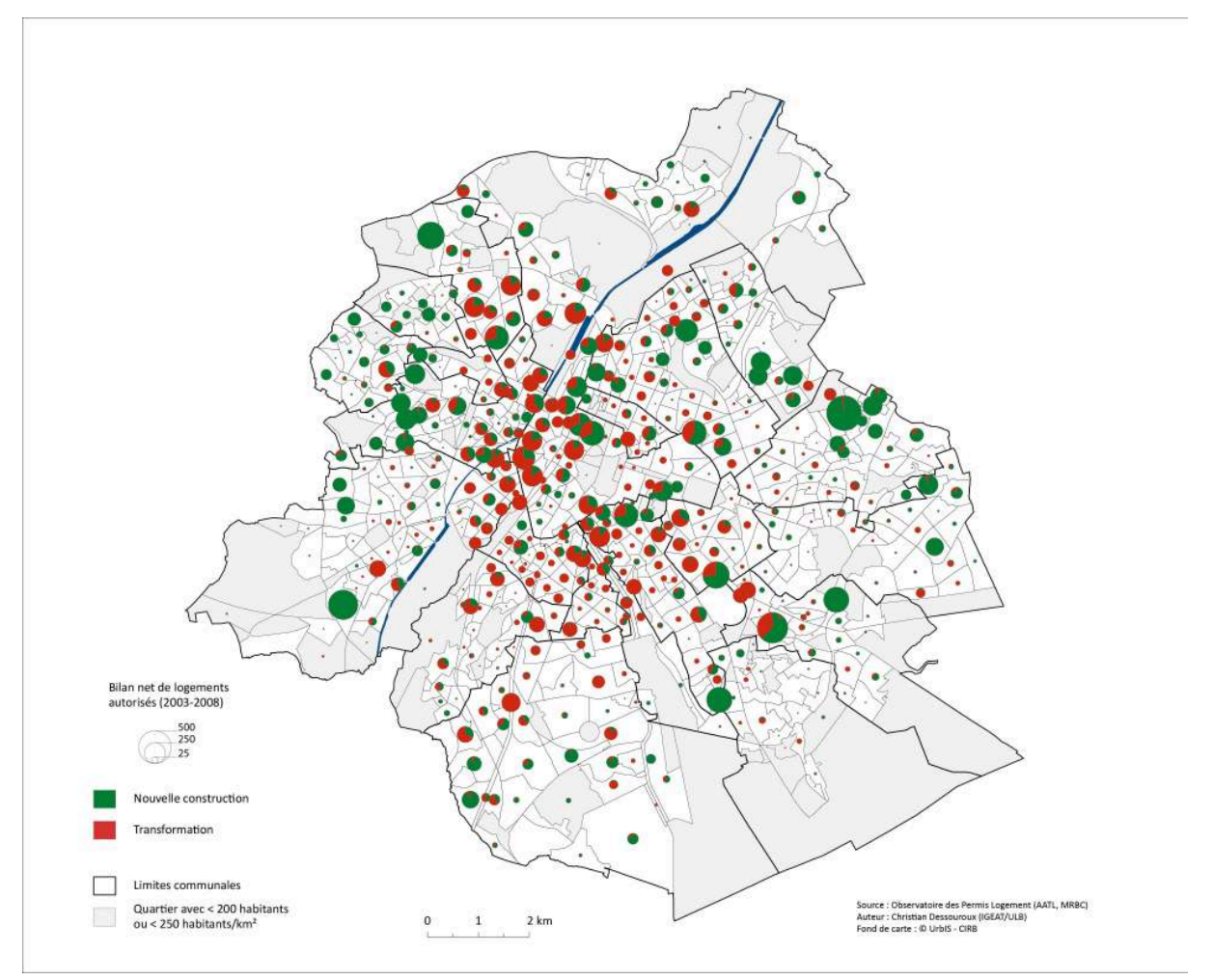

Source : Observatoire des Permis Logement (AATL, MRBC) - Cartographie : Ch. Dessouroux; fond de carte : (c) urbIS-CIRB.

La carte (illustration 7) donne à voir un niveau de production résidentielle relativement soutenu dans l'ensemble de la Région bruxelloise et plus particulièrement dans les quartiers centraux et aux extrémités de l'espace bâti. Le parc de logements a grandi d'environ 5000 unités par an entre 2003 et 2008, ce qui donne un taux d'accroissement 
moyen de $1,23 \%$. Les taux les plus élevés se rencontrent à proximité des boulevards formant le "pentagone " central bruxellois (le long du canal, dans les environs de la gare du Nord, dans le nord d'Ixelles et à proximité du quartier européen), ainsi que ponctuellement dans les communes les plus excentriques disposant d'importantes réserves foncières constructibles.

Les taux de transformation sont particulièrement élevés dans les zones centrales offrant peu d'espace de développement pour les constructions nouvelles (hormis la viabilisation des friches urbaines et les opérations de démolition-reconstruction), mais disposant d'un important stock de bâtiments industriels, d'entrepôts, d'ateliers, ainsi que de bureaux vacants, susceptibles d'une reconversion moyennant transformation.

Illustration 8 - Reconversions en logement à Bruxelles, par quartier (2003-2008)

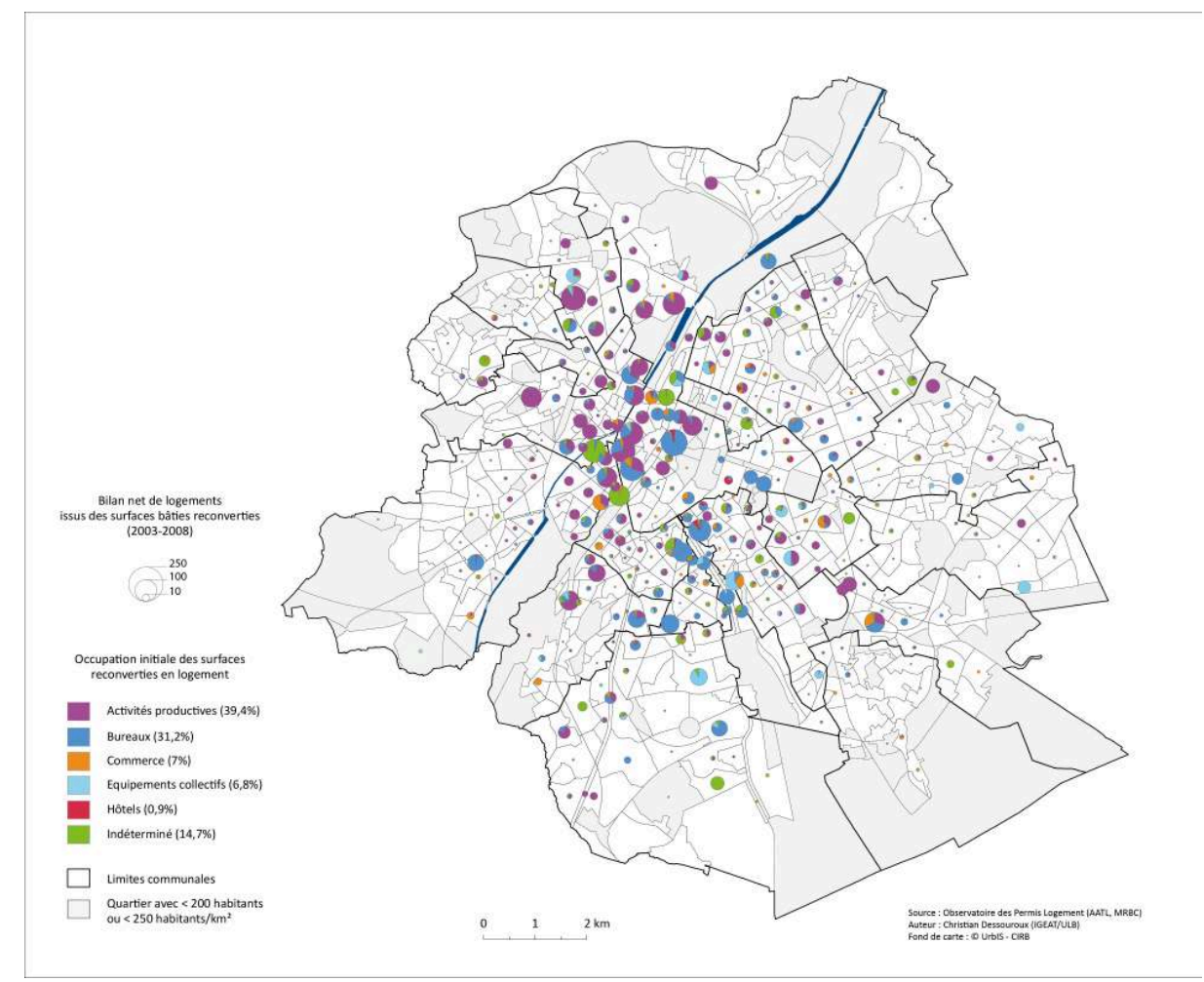

Source : Observatoire des Permis Logement (AATL, MRBC) - Cartographie : Ch. Dessouroux; fond de carte : (c) urbIS-CIRB.

La géographie des reconversions de bâtiments (ou parties de bâtiments) non résidentiels en logements se recouvre évidemment avec celle des transformations (illustration 8). Quasi inexistant encore dans les années 1980, ce phénomène gagne du terrain. Il concerne avant tout l'ouest de la ville centrale, disposant d'un important stock de bâtiments industriels dans les quartiers ouvriers le long du canal, ainsi que les quartiers résidentiels à l'est et au sud-est du centre-ville où nombre de bureaux avaient un temps occupé (et occupent toujours) des immeubles destinés au logement. On observe par ailleurs, de manière plus ponctuelle, d'importantes opérations de reconversion concernant des commerces ou des équipements collectifs. Dans l'ensemble, ces projets de reconversion, constituant une part importante de la production globale, reflètent à la fois la dynamique récente de rénovation des quartiers 
centraux anciens, mais également la nécessaire densification du tissu résidentiel face à la demande croissante de logements.

Si la densification urbaine est désormais valorisée et recherchée (Declève et al., 2009), elle ne peut toutefois pas se faire sans provoquer de tensions. L'offre en espace habitable n'est en effet pas extensible à volonté. Elle est limitée à la fois par les réserves foncières disponibles et le degré de tolérance des riverains à la densification qui fragiliserait, selon eux, la qualité de leur cadre de vie. La question de la densité de l'espace urbain illustre dès lors les mutations en cours dans les agglomérations, mais révèle aussi les contradictions qu'elle soulève : à la fois offrir plus de logements et préserver des espaces verts, amener de nouveaux habitants et réduire la circulation automobile....

\section{Les producteurs de logements}

Les données collectées par l'Administration régionale donnent, pour chaque permis d'urbanisme ${ }^{10}$ délivré entre 2003 et 2008, en plus des informations détaillées concernant les travaux autorisés, l'identité et l'adresse du demandeur de permis ${ }^{11}$. Ces informations permettent d'identifier et d'étudier séparément les différentes catégories de producteurs de logement.

A Bruxelles, la production de logements est aujourd'hui principalement le fait de sociétés de promotion immobilière : $70 \%$ des créations de logements entre 2003 et 2008 se font à travers des permis d'urbanisme délivrés à des sociétés privées (voir le tableau en illustration 9 - seules les créations de logements sont prises en compte, pas les suppressions). Cette prépondérance des sociétés est propre à la Région bruxelloise et ne se retrouve pas, du moins au niveau régional, en Flandre et en Wallonie (Dubois, 2002).

Si beaucoup d'observateurs s'accordent sur l'idée que le marché immobilier bruxellois s'est internationalisé ces dernières années, notamment à travers les interventions des filiales Real Estate des grandes banques et la création en 1995 d'un statut spécial pour les Fonds d'Investissement en Immobilier (SICAFIs) belges, les chiffres ont manqué jusqu'à ce jour pour objectiver ce phénomène. Les petites sociétés de promotion indépendantes, constituées par des particuliers et dont l'actionnariat reste individuel ou familial, sont en tout cas encore très présentes dans le secteur de la promotion résidentielle à la fin des années 2000. Les sociétés prises dans leur ensemble créent en moyenne 10 logements par opération immobilière, ce qui indique que la promotion immobilière reste une entreprise profitable même lorsqu'elle porte sur de très petits projets.

Illustration 9 - Nombre de logements autorisés par type de producteur (2003-2008)

\begin{tabular}{|l|l|l|}
\hline Type de producteur & $\begin{array}{l}\text { Nombre de logements autorisés (permis } \\
\text { d'urbanisme délivrés entre 2003 et 2008 - } \\
\text { création de logements seulement) }\end{array}$ & $\begin{array}{l}\text { Part dans le total } \\
\text { autorisés }\end{array}$ \\
\hline Sociétés privées & 20070 & $70 \%$ \\
\hline Particuliers (Bailleurs) & 4280 & $15 \%$ \\
\hline
\end{tabular}




\begin{tabular}{|l|l|l|}
\hline Particuliers (Occupants) & 1290 & $4 \%$ \\
\hline Institutions publiques & 2020 & $7 \%$ \\
\hline Partenariats public-privé12 & 860 & $3 \%$ \\
\hline $\begin{array}{l}\text { Autres (associations, } \\
\text { seniories, établissements } \\
\text { scolaires, ...) }\end{array}$ & 210 & $1 \%$ \\
\hline
\end{tabular}

Source : Observatoire des Permis Logement (AATL, MRBC).

47 Si l'on fait l'hypothèse qu'il n'y a pas de promoteur professionnel sans société - la constitution d'une société présentant des avantages financiers et juridiques - et que la propriété bailleresse est, elle, essentiellement le fait de particuliers (Charles, 2007), les particuliers qui mènent des opérations immobilières sont soit les occupants (ou les futurs occupants) des logements créés, soit ils les créent pour les mettre en location. Nous avons assimilé à des «occupants » les particuliers menant des opérations de 1 ou 2 logements, et à des «bailleurs » lorsque le nombre résultant de logements dépassait 2. Selon cette classification, $15 \%$ des logements sont produits par des bailleurs, et une très petite partie $(4 \%)$ des logements est produite par leurs futurs occupants.

Quelques $7 \%$ des logements créés entre 2003 et 2008 le sont à travers des permis d'urbanisme octroyés à des structures dépendant des pouvoirs publics (régionaux ou communaux) ; la majorité sont des logements sociaux, réservés aux ménages dont les revenus ne dépassent pas 20.000 euros par $a^{13}$. Au nom de la mixité sociale, les opérations publiques incluent cependant de plus en plus souvent une certaine proportion de «logements moyens », accessibles aux ménages ayant des revenus plus élevés.

$493 \%$ des logements produits sont des logements "conventionnés », produits par des partenariats public-privé à l'initiative de la Société de Développement pour la Région de Bruxelles-Capitale (SDRB). Ces partenariats ont pour but d'attirer les investisseurs privés dans certains quartiers en leur garantissant une rentabilité suffisante via un subside. L'intervention publique permet également de vendre ces logements à des prix inférieurs à ceux du marché. Les partenaires privés de la SDRB sont généralement de grosses sociétés de promotion immobilière, très présentes par ailleurs sur le marché bruxellois. Les logements produits par la SDRB sont accessibles aux ménages dont les revenus sont inférieurs à 55000 euros par an ${ }^{14} c^{\prime}$ est-à-dire, en réalité, à la majorité des ménages bruxellois ( $90 \%$ des ménages bruxellois d'une personne, par exemple, satisfont à ces conditions de revenu).

\section{Stratégies spatiales des investisseurs}

La proportion des logements produits (autorisés par les permis d'urbanisme) par les différents types d'acteurs a été analysée quartier par quartier (illustration 10), ce qui permet de comparer leurs stratégies spatiales. 


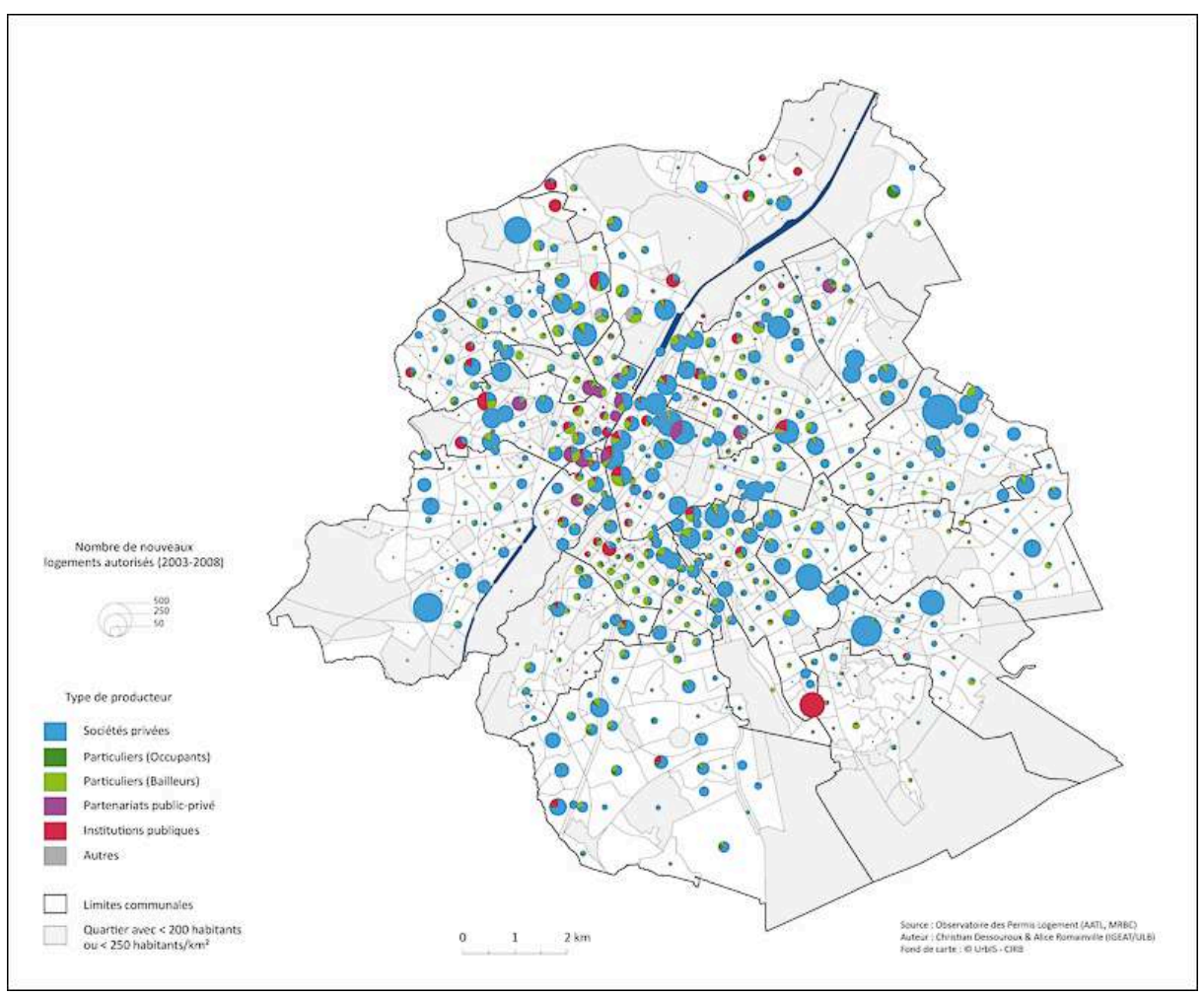

Source : Observatoire des Permis Logement (AATL, MRBC) - Cartographie : Ch. Dessouroux et A. Romainville; fond de carte : (c) urbIS-CIRB.

Les sociétés sont présentes partout dans la Région. Sans surprise, elles sont pratiquement hégémoniques dans les quartiers où les permis accordés concernent de grosses opérations, c'est-à-dire dans les espaces les plus périphériques à Woluwe-SaintLambert, Anderlecht, Molenbeek... (constructions neuves principalement), mais aussi dans certains quartiers plus centraux (quartier européen, environs de la gare du Nord, par exemple), où l'on trouve également de grosses opérations de transformation/ reconversion.

La part des particuliers parmi les producteurs de logements est surtout importante à Saint-Gilles et dans certains quartiers d'Anderlecht, de Molenbeek et des communes aisées de l'est de la Région. A Saint-Gilles et à Molenbeek, ce sont surtout des propriétaires bailleurs. Ceux-ci produisent, dans certains quartiers, plus de la moitié des logements. Les opérations menées par des bailleurs sont généralement petites (2-3 logements créés en moyenne). Il faut rappeler ici que beaucoup d'opérations de rénovation - et même certaines opérations de construction - sont menées de façon illégale sans permis d'urbanisme, ce qui a entre autres comme conséquence que la production de logements par les propriétaires à leur propre domicile est très largement sous-estimée par les chiffres présentés ici. Charles (2007) a montré que les bailleurs à domicile sont très présents dans le « croissant pauvre » bruxellois, où près de $50 \%$ des propriétaires occupants seraient par ailleurs bailleurs à domicile.

Charles (2007) a par ailleurs montré que les propriétaires bailleurs ont des stratégies spatiales distinctes selon la taille de leur portefeuille immobilier: les bailleurs de standing élevé et possédant un portefeuille immobilier important sont surreprésentés dans le pentagone et dans le sud-est de la première couronne (haut de Saint-Gilles et de 
Forest, Ixelles, Etterbeek), là où l'intensité des déménagements est la plus élevée (Deboosere et al., 2009, p. 4). Dans ces quartiers, les bailleurs compensent le risque lié à la mobilité des locataires par un patrimoine plus important et par la possibilité de revaloriser plus régulièrement (à chaque changement de locataire) les loyers qu'ils pratiquent.

Les producteurs publics sont surtout présents en seconde couronne avec une série de projets de logements sociaux (dont le plus important est le projet Ernotte, à la frontière entre Ixelles et Watermael-Boitsfort, avec 234 logements sociaux et 82 logements moyens). Les institutions publiques sont aussi présentes dans le centre, avec des projets associant souvent logements sociaux, logements locatifs "moyens» et logements acquisitifs " conventionnés ", principalement dans le cadre des " contrats de quartier ", des programmes de "revitalisation » intensive ciblés sur les quartiers populaires du centre.

Les logements créés par les partenariats public-privé, toujours sur la période 2003-2008, sont très concentrés le long du canal, principalement dans la commune de Molenbeek. Le périmètre d'action de la SDRB a en effet été circonscrit précisément: elle investit les quartiers populaires du centre qui sont la cible du vaste projet de « revitalisation » mené par le pouvoir régional. Cette branche de la politique régionale de l'habitat, mise en place dans les années 1990, qui associe promotion de la propriété privée et rénovation urbaine, représente environ la moitié du budget régional consacré à la politique de l'habitat (Zimmer, 2006). Elle a comme objectif principal de restaurer l'attractivité résidentielle de la Région pour les ménages à revenus moyens et supérieurs, dont le retour (ou le maintien) en ville est censé à la fois contribuer à la rénovation du bâti, redresser les finances régionales via leur contribution fiscale, et «favoriser la mixité sociale »- dans les quartiers pauvres s'entend ${ }^{15}$.

56 A travers différents outils (subsides aux investisseurs privés, rénovation des espaces publics, primes et abattements fiscaux pour les propriétaires, règlements en matière de commerce de détail...), la Région canalise donc l'investissement privé vers les zones qu'elle aimerait voir réinvesties, à la fois par les capitaux et par une population plus aisée. Les pouvoirs publics jouent le rôle d'initiateurs et/ou de garants pour certaines opérations de rénovation et de cette façon, structurent le déploiement spatial du processus de gentrification (Romainville, 2010). Les hausses de prix générées par ces investissements publics et privés ne sont pas en mesure d'être freinées par les interventions publiques sur le marché du logement proprement dit: ce marché reste complètement dérégulé, et comme on l'a vu, la " part de marché » des pouvoirs publics n'atteint pas les $10 \%$.

\section{Conclusion}

Le traitement des données sur les permis de bâtir a permis de brosser un portrait du marché du logement en Belgique et à Bruxelles, et de souligner certaines spécificités belges et évolutions récentes. Il en ressort que l'intervention - ou l'absence d'intervention - des pouvoirs publics est, de façon assez systématique, un facteur explicatif majeur des géographies et évolutions temporelles observées.

Historiquement, la philosophie libérale du code civil belge et la longue hégémonie des partis chrétiens au gouvernement ont déterminé une protection forte du droit de propriété individuelle, très peu de régulation dans le secteur locatif et une politique du 
logement fortement axée sur la promotion de la propriété privée (De Decker, 2001). Cette « aide à la pierre » intensive et beaucoup de laxisme dans l'affectation des sols ont déterminé une très grande proportion de propriétaires, un grand succès de l'autopromotion, et une périurbanisation sous forme de maisons individuelles, phénomène massif qui est loin d'être terminé, assurant un étalement urbain particulièrement lâche - et problématique sous plusieurs aspects. Pour le reste, la géographie de la production de logements et son évolution reflètent les principales évolutions démographiques du pays.

Parmi les évolutions récentes les plus notables, il faut souligner la diminution de l'intervention publique sur le secteur locatif, jusqu'à devenir aujourd'hui pratiquement négligeable, que ce soit en termes de régulation du marché ou de production de logements. Autre évolution, et ce surtout dans les régions urbaines et en Wallonie, la place croissante prise par les rénovations dans la production de logements. A Bruxelles, une partie de ces rénovations sont des reconversions de bâtiments fonctionnels en logements. A nouveau, les mesures mises en place par les pouvoirs publics expliquent au moins en partie cette croissance. Ces aides à la rénovation participent, à côté des aides à l'acquisition, à l'intensification de l' "aide à la pierre " s'adressant aux propriétaires et dont l'analyse a montré, à Bruxelles, qu'elles ne sont pas neutres socialement et qu'elles jouent un rôle dans les processus de ségrégation socio-spatiale.

Les chiffres montrent également la part croissante des sociétés sur le marché du logement, phénomène qui n'est pas sans lien avec la production croissante de logements par reconversion ou rénovation du bâti ancien. A Bruxelles, la présence importante des sociétés de promotion sur le marché s'explique, au moins en partie, par les possibilités accrues de plus-values qu'offrent les dynamiques de revalorisation foncière et de gentrification - le rôle des politiques publiques est ici, encore une fois, tout à fait déterminant.

\section{BIBLIOGRAPHIE}

Ananian P., 2010. La production résidentielle comme levier de la régénération urbaine à Bruxelles. Louvain-la-Neuve, Presses universitaires de Louvain, 320 p.

Biot V., Van Hamme G., 2007. Impact socio-économique de la présence des institutions de l'Union européenne et des autres institutions internationales en Région de Bruxelles-Capitale. Eléments de mesure. Bruxelles, IGEAT, Université libre de Bruxelles (rapport de recherche inédit), 34 p.

Capron C., Eggerickx T., Hermia J.-P., 2002. Les nouvelles dynamiques de peuplement dans les zones frontalières en Wallonie. Espace populations sociétés, 1-2, p. 83-98.

Charles J., 2006. From poor tenants to resident landlords: de facto social housing homeownership in Brussels. Communication au colloque "Home Ownership in Europe: Policy and Research Issues", European Network for Housing Research, Delft, 23-24 novembre 2006.

Charles J., 2007. Structure de la propriété sur le marché locatif privé bruxellois (rapport inédit). Bruxelles, Prospective Research for Brussels, 106 p. 
Deboosere P., Eggerickx T., Van Hecke E., Wayens B., 2009. La population bruxelloise : un éclairage démographique. Note de synthèse $n^{\circ} 3$ des Etats Généraux de Bruxelles. Brussels Studies (disponible sur www.brusselsstudies.be), 17 mars 2009, 17 p.

Declève B. (dir.), Ananian P., Anaya M., Lescieux A., 2009. Densités bruxelloises et modèles d'habiter. Région de Bruxelles-Capitale, $308 \mathrm{p}$.

De Decker P., 2001. Jammed between housing and property rights: Belgian private renting in perspective. European Journal of Housing Policy, n 1 (1), p. 17-39.

De Lannoy W., Kesteloot C., 1990. Les divisions sociales et spatiales de la ville. In : Mort-Subite, "Les fractionnements sociaux de l'espace belge : une géographie de la société belge", Contradictions, $n^{\circ}$ 58-59, p. 153-190.

Doucet P., 1983. Le déclin de la petite propriété bailleresse. Courrier hebdomadaire du CRISP, $\mathrm{n}^{\circ}$ 995, $32 \mathrm{p}$.

Dubois O., 2002. Stratégies des acteurs de la construction résidentielle neuve et caractéristiques des espaces bâtis en Belgique. Belgeo, 4, p. 319-332.

Dubois O., 2004. Fiscalité immobilière résidentielle et développement urbain. Un état des lieux du rôle du précompte immobilier comme outil de développement de la Région bruxelloise. Rapport après 2 ans, Région de Bruxelles-Capitale, Prospective Research for Brussels, $123 \mathrm{p}$.

Eggerickx T., Sanderson J.-P., Bahri A., Hermia J.-P., 2007. Diversités démographiques et migrations résidentielles dans les espaces ruraux en Wallonie. Norois, 205, p. 117-136.

Goossens L., Thomas I., Vanneste D., 1999. Recensement général de la population et des logements au 1er mars 1991. Monographie $N^{\circ} 10$ - Le logement : réalités socio-économiques et géographiques 1981-1991. Bruxelles, Institut national de Statistique, $310 \mathrm{p}$.

Gouvernement wallon, 1999. Schéma de Développement de l'Espace Régional (SDER). Namur (disponible sur : http://sder.wallonie.be ).

Halleux J.-M., Brück L., Mairy N., 2002. La périurbanisation résidentielle en Belgique à la lumière des contextes suisse et danois : enracinement, dynamiques centrifuges et régulations collectives. Belgeo, 4, p. 333-354.

Halleux J.-M, 2005. Le rôle des promotions foncières et immobilières dans la production des périphéries : application à la Belgique et à ses nouveaux espaces résidentiels. Revue Géographique de l'Est, $\mathrm{n}^{\circ}$ 3-4, p. 161-174.

Kesteloot C., de Turck A., Vandermotten C.. et al., 2001. Structures sociales et quartiers en difficulté dans les régions urbaines belges. Sociale structuren en buurten in moeilijkheden in de Belgische stadsgewesten,KULeuven et ULB, Politique des grandes villes, $108 \mathrm{p}$.

Louvot C., 2001. Le logement dans l'Union européenne : la propriété prend le pas sur la location. Economie et statistique, 343 (1), p. 29-50.

Mougenot C., 1988. Promoting the single-family house in Belgium. The social construction of model housing. International Journal of Urban and Regional Research, $\mathrm{n}^{\circ} 12$ (4), p. 531-549.

Noël F., 1998. La ville rapiécée. Les stratégies de la réhabilitation à Bruxelles. Bruxelles, Editions de l'Université Libre de Bruxelles, 189 p.

Noël F., 2009. Logements et habitat s'exposent à l'Exposition Universelle de 1958. In : Deligne C., Jaumain S. (dir.), L'expo 58. Un tournant dans l'histoire de Bruxelles. Bruxelles, Le Cri, Histoire, p. 145-183. 
Observatoire de la santé et du social, 2009. Baromètre social. Rapport bruxellois sur l'état de la pauvreté. Bruxelles, Commission Communautaire Commune, 84 p. (disponible sur http:// www.observatbru.be ).

Romainville A., 2007. Une géographie de la pression fiscale pesant sur les ménages dans la région urbaine bruxelloise, $17^{7 \mathrm{me} e}$ Congrès des Economistes belges de Langue française : « Nos Régions en Europe », CIFoP, Louvain-La-Neuve (disponible sur http://igeat.ulb.ac.be ).

Romainville A., 2010. A qui profitent les politiques d'aide à l'acquisition de logements à Bruxelles ?Brussels Studies (disponible sur www.brusselsstudies.be), 34, 19 p.

Surkyn J., Willaert D., Marissal P., Charles J., Wayens B., 2007. La Région de Bruxelles-Capitale face à son habitat : étude structurelle et prospective. Rapport inédit pour madame F. Dupuis, secrétaire d'Etat au logement de la Région de Bruxelles-Capitale, 175 p.

Topalov C., 1974. Les Promoteurs immobiliers. Contribution à l'analyse de la production capitaliste du logement en France. Paris, La Haye-Mouton, 417 p.

Van Criekingen M., Decroly J-M., 2003. Revisiting the diversity of Gentrification: Neighbourhood Renewal Processes in Brussels and Montreal. Urban Studies, vol. 40, 12, p. 2451-2468.

Van Criekingen M., 2006. Que deviennent les quartiers centraux à Bruxelles ?Brussels Studies (disponible sur www.brusselsstudies.be), 1, $21 \mathrm{p}$.

Van Criekingen M., 2009. Moving in/out of Brussels' historical core in the early 2000s: migration and the effects of gentrification, Urban Studies, 46/4, p. 825-848.

Vandermotten C., 2003. La navette de travail vers Bruxelles : des abonnements ouvriers au RER. In Laconte P. (dir.), La gare et la ville. Grands axes et réseau express régional : enjeux et perspectives. Bruxelles, Publication de la Fondation pour l'Aménagement Urbain, p. 49-57.

Willems P., 2008. Migratiebewegingen in het Vlaamse Gewest in de periode 1997-2006. SVR-Rapport, 2. Studiedienst van de Vlaamse Regering, $57 \mathrm{p}$.

Zimmer P., 2006. Politique du logement en Région de Bruxelles-Capitale. Les échos du logement (disponible sur http://mrw.wallonie.be ), 2, p. 4-15.

\section{NOTES}

1. Estimation pour 2008 sur base du nombre de ménages (Source: DGSIE). Le Cadastre (Administration Générale de la Documentation Patrimoniale) fournit un chiffre comparable pour 2008 (4,7 millions de logements), mais tient également compte des logements non occupés.

2. Source : DGSIE, Statistiques de l'occupation du sol et du cadastre (1995).

3. Plus particulièrement par la Direction Urbanisme de l'Administration de l'Aménagement du Territoire et du Logement (AATL) au Ministère de la Région de Bruxelles-Capitale.

4. Le Ruimtelijke Structuurplan Vlaanderen (RSV) en Flandre (1997) et le Schéma de Développement de l'Espace Régional (SDER) en Wallonie (1999) constituent des documents d'orientation qui annoncent des ambitions, mais qui n'ont pas de valeur réglementaire. Les documents réglementaires, en particulier les plans de secteur qui ont été adoptés dès les années 1970 à un moment où la politique restrictive d'utilisation du sol n'était pas encore de mise, font preuve d'inertie par rapport aux orientations de ces schémas.

5. Les «plans de secteur" réglementaires, élaborés dès les années 1960 à un moment où la politique restrictive n'était pas encore de mise, font notamment preuve d'inertie par rapport aux orientations indicatives et volontaristes, mais non réglementaires, des schémas régionaux. 
6. Dans un très grand nombre de communes et de villes wallonnes, plus de $40 \%$ du parc immobilier est constitué de logements construits avant 1945. Plus d'un quart de tous les logements wallons actuels ont été construits avant 1945, comparé à $15 \%$ pour la Belgique (Source : DGSIE, Enquête socio-économique 2001).

7. Il était de $27 \%$ en 1971 , de $31 \%$ en 1981 , de $40 \%$ en 1991 et de $41 \%$ en 2001 (d'après les recensements INS).

8. Logements où il manque au moins une chambre, le nombre de chambres nécessaires étant calculé sur base de la composition des ménages (selon les normes du logement social) (source: enquête socio-économique 2001, DGSIE).

9. La " plateforme NOVA » (application informatique de soutien à l'instruction des demandes de permis d'urbanisme) gérée par la Direction Urbanisme de l'Administration de l'Aménagement du Territoire et du Logement (AATL) centralise l'ensemble des permis d'urbanisme. L'Observatoire des permis logement, géré par la Direction Etudes et Planification, assure une exploitation statistique de ces données.

10. Terme utilisé en Région de Bruxelles-Capitale pour les permis de bâtir.

11. Ce demandeur est logiquement, dans la majorité des cas, le propriétaire du bien, même si légalement le permis peut être demandé par quelqu'un d'autre.

12. Logements produits par la Société de Développement pour la Région de Bruxelles-Capitale uniquement.

13. Conditions en 2010. Ce plafond varie selon le nombre de revenus et de personnes à charge dans le ménage.

14. Conditions en 2010. Ce plafond varie selon le nombre de revenus et de personnes à charge dans le ménage.

15. Voir le Plan Régional de Développement arrêté en 2002, http://www.prd.irisnet.be

\section{RÉSUMÉS}

Le paysage résidentiel belge s'est transformé considérablement depuis les années 1950. Si l'activité économique et le logement collectif continuent à se concentrer dans les agglomérations urbaines, la dispersion de l'habitat individuel dans les zones périurbaines, entamée au $19^{\mathrm{e}}$ siècle, se poursuit sans relâche. D'autres phénomènes sont plus contemporains, comme la réhabilitation et la reconversion croissantes du bâti existant, alimentées parmi d'autres par une pénurie de plus en plus aigue de logements et une revalorisation du patrimoine immobilier ancien.

Le présent article offre un panorama de la production résidentielle en Belgique depuis les années 1950 mais étudie plus particulièrement les évolutions récentes à partir de sources statistiques peu exploitées à ce jour. Moyennant une analyse d s permis de bâtir délivrés depuis 1996, nous essayons de mettre en évidence les spécificités du contexte belge et les changements récents les plus significatifs en termes de production de logements, tant sur le plan quantitatif que géographique. L'importance respective des organismes publics et des différents acteurs privés sur le marché résidentiel sera étudiée en s'appuyant sur l'exemple de la Région bruxelloise, pour laquelle des données très précises ont été exploitées.

The Belgian residential landscape has considerably changed since the 1950s. If economic activity and collective housing continue to be concentrated in urban areas, the proliferation of singlefamily dwellings in suburban areas - dating back to the 19th century - continues unabated. Other 
developments are more recent, such as an increase in refurbishments and conversions of existing buildings, fuelled by among other things the insufficient production of new housing and a revaluation of older housing.

This article provides an overview of residential construction in Belgium since the 1950s, focusing particularly on more recent changes using statistical resources that have yet to be fully explored. Through an analysis of building permits issued since 1996, we will try to highlight the particularities of the Belgian context and to reveal the most significant changes in terms of housing production, both quantitatively and geographically. The relative importance of public and private agents on the housing market will be studied using the example of the Brussels Region, for which very accurate data has been used.

\section{INDEX}

Keywords : Belgium, Brussels, construction, conversion, housing

Mots-clés : Belgique, Bruxelles, construction, logement, transformation

\section{AUTEURS}

\section{CHRISTIAN DESSOUROUX}

Christian Dessouroux (cdessour@ulb.ac.be) est docteur en géographie et chercheur postdoctoral Prospective Research for Brussels à l'Institut de Gestion de l'Environnement et Aménagement du Territoire de l'Université libre de Bruxelles (ULB). Il a travaillé sur le rôle des espaces publics dans les politiques urbaines contemporaines avant de publier une recherche sur le développement urbanistique de Bruxelles au $19^{\mathrm{e}}$ et $20^{\mathrm{e}}$ siècle (Espaces partagés, espaces disputés. Bruxelles, une capitale et ses habitants). Il travaille actuellement sur l'offre résidentielle à Bruxelles dans le contexte des processus de croissance démographique et de dualisation sociale.

\section{ALICE ROMAINVILLE}

Alice Romainville (alice.romainville@ulb.ac.be) a mené, comme géographe, plusieurs recherches à l'Institut de Gestion de l'Environnement et Aménagement du Territoire (ULB), portant notamment sur la démographie et la géographie économique en Belgique, la fiscalité et les politiques de logement en Région bruxelloise. Ses recherches actuelles, au département de Géographie de l'ULB où elle est assistante, portent sur l'offre de logements et la promotion immobilière à Bruxelles. 